\title{
LA POBREZA COMO ESPACIO DE INDETERMINACIÓN Un análisis desde la biopolítica
}

\author{
POVERTY AS SPACE OF INDETERMINATION
}

An Analysis from Biopolitics

\author{
Nelson Arteaga Botello \\ Universidad Autónoma del Estado de México \\ arbnelson@yahoo.com \\ María Luisa Bacarlett Pérez \\ Universidad Autónoma del Estado de México \\ cioran6472@yahoo.com
}

\section{Resumen}

En este artículo se analiza el problema de las políticas que atienden la pobreza como una forma particular de biopolítica de la población, con el fin de resaltar la contradicción que generan al propiciar tanto la inclusión como la exclusión de los sectores considerados como pobres. Dicha contradicción se analiza desde tres ejes: la relación jurídica que se establece con la figura del pobre, como esto define un espacio de indeterminación de la población pobre y, finalmente, las implicaciones que ello tiene en la conformación de una vigilancia específica sobre estos sectores sociales. De esta manera se observa que las políticas de atención a la pobreza se fundan en una estrategia que maximiza las vida de las poblaciones depauperadas al mismo tiempo que tiende a mantenerla en niveles de supervivencia. Aspecto al que habría que prestar mayor atención en los efectos que pudiera tener en la consolidación democrática de la región.

\section{Palabras Clave}

Control social, Estado de excepción, Necropolítica, Vida nuda, Vigilancia.

\section{ABstRact}

In this article is analyzed the problem posed by policies that handle poverty as a particular way of biopolitics of population with the purpose of enhancing the contradiction generated by favouring the inclusion as well as the exclusion of the sectors considered as poor. Such contradiction is analyzed from three different edges: the legal relation established with the figure of the poor, how it defines a space of indetermination of poor population and finally the implications it has in the conformation of a specific surveillance of these social sectors. In this way it is observed that the poverty attention policies are based in a strategy which maximizes the lifes of the de-poverted populations white it tends to maintain them in surviving levels. Considering the effects it may have on the democratic consolidation of the region, it slanld be payed more attetion to this issue.

\section{KEYWORDS}

Bare Life, Necropolitics, Social Control, State of Exception, Surveillance. 


\section{INTRODUCCIÓN}

En los últimos años, tras su incremento, las políticas neoliberales se han hecho acompañar por la puesta en marcha de estrategias orientadas a frenar sus efectos más perniciosos, entre ellos -y quizás el principal- la pobreza. A lo largo y ancho de la región latinoamericana se han diseñado y puesto a andar distintas estrategias, trazadas muchas de ellas por las propias oficinas donde se maquilaron las políticas de choque económico, buscando dotar a la población de alimento, empleo, recursos económicos, capacitación, entre otros elementos considerados como detonadores necesarios para salir de la pobreza (Duffield, 2002; Sandbrook y Romano, 2004). La mayoría de las veces estas políticas son definidas por los académicos más críticos como meros paliativos, mientras que sus defensores esgrimen "evidencia" de casos muy concretos para demostrar la efectividad de dichas políticas.

Sin embargo, consideramos que estas dos posiciones pasan de largo otra directriz que nos permitiría relacionar el problema de la pobreza con la constitución de una "biopolítica de la población" por parte de los estados contemporáneos latinoamericanos, reparando en el cuerpo-especie, en la sociedad, como un todo sobre el cual tratan de controlarse los procesos biológicos: muertes, nacimientos, enfermedades, duración de la vida, condiciones de salud y de vida, etc. (Alvarez 2005; Bialakowski 2007). Recordemos que desde la perspectiva foucaultiana, la vida se convierte en un affaire que se trata de optimizar, de maximizar, es decir, "un poder destinado a producir fuerzas, a hacerlas crecer y a ordenarlas más que a desaparecerlas, a hacerlas plegar o destruirlas" (Foucault 1976: 179). En el caso de las sociedades liberales, en particular, apunta este autor, la vida se convierte en una realidad sobre la que se ha de trabajar positivamente: administrándola, optimizándola, ordenándola. Este poder ejercido positivamente sobre la vida será incluyente y normalizador: antes que excluir y borrar del mapa, incluye en un sistema de orden y normalización.

Siguiendo la propuesta foucaultiana, la biopolítica se vincula fundamentalmente a las sociedades liberales, las cuales son, de hecho, el "cuadro general de la biopolítica". Pero si bien la biopolítica pareciera actuar en un sentido positivo -ya que se trata, ante todo, de gestionar y maximizar las fuerzas vitales con miras a su conducción y utilización-, ello no descarta que este mismo poder despliegue gestos negativos y excluyentes, pues si el objetivo es mejorar la vida, entonces habrá que excluir o abolir todo aquello que vaya contra esta maximización de la vida. En las llamadas políticas contra la pobreza parecen encontrarse estos dos elementos de la biopolítica: el intento por maximizar la vida de los sectores empobrecidos, como también el de excluirlos, dejarlos morir, de alguna forma, conformando una especie de necropolítica (Mbembe 2003; Sylvester 2005).

El presente documento tiene como objetivo mostrar cómo el problema de la pobreza es un punto de intersección de estrategias biopolíticas desplegadas por el Estado, a través de las cuales se puede observar esta contradicción entre el cuidado de la vida de aquellos que son considerados como pobres, así como su exclusión. Dicha contradicción se puede observar en tres momentos. El primero, refiere a la relación jurídica que 
el Estado establece con la figura del pobre a través de las políticas públicas diseñadas para combatir la pobreza. El segundo, derivado del anterior, trata de la construcción de un espacio de indeterminación del sujeto pobre que lo pone de alguna forma como figura de la "vida nuda" (Agamben 2006) ${ }^{1}$. Finalmente, el necesario papel de la vigilancia para la delimitación de esta figura, que implica que a través de distintos dispositivos el Estado vigile, controle y gestione los movimientos, comportamientos y acciones de los pobres, instalando con ello un tipo particular de gestión de la población. El análisis de estos tres momentos, consideramos, permitiría pensar de una forma distinta las estrategias de "lucha contra la pobreza", visualizándolas como una expresión del ejercicio biopolítico del Estado neoliberal en América Latina, el cual se encuentra generando una serie de dispositivos de gubernamentalidad y subjetivización sobre los que habría de prestar mayor atención, así como considerar sus efectos en la llamada consolidación democrática de la región.

\section{DISPOSITIVOS DE VIGILANCIA: PROTECCIÓN Y CONTROL DE LOS POBRES}

En su libro Vigilar y castigar, Foucault analiza los procesos a través de los cuales son constituidos el cuerpo y el alma de los individuos y grupos sociales modernos, sobre todo a partir de instituciones disciplinarias cerradas como el ejército, la escuela y el hospital. No obstante, para este autor la disciplina solamente es la expresión de una lógica más amplia: la vigilancia.

Efectivamente, el poder disciplinario se ejerce a partir de la capacidad de vigilar a aquellos que se busca corregir: "los locos, los niños, los escolares, los colonizados, sobre todos aquellos que se han fijado en un aparato de producción y que se necesita controlar a todo lo largo de su existencia" (Foucault 1975: 38). El éxito del poder disciplinario radica en el uso de instrumentos como la mirada jerarquizada, a la que se suma la sanción normalizadora y la aplicación del examen. Esta vigilancia no responde simplemente a la necesidad de disciplinar. Por el contrario, la observación y la mirada atentas constituyen expresiones de un aparato donde las técnicas que potencian la visión inducen efectos de poder, coerción y control. Foucault resalta cómo, al mismo tiempo que se construyen lentes y lentillas para microscopios y telescopios, se crean

[...] pequeñas técnicas de vigilancias múltiples y entrecruzadas, de miradas que deben ver sin ser vistas, un arte oscuro de luces y de lo visible ha preparado en silencio un saber nuevo del hombre a través de técnicas para su dominación y de procedimientos para su utilización (Foucault 1975: 201)

${ }^{1}$ Cabe señalar que para Agamben, a diferencia de Foucault, la biopolítica no es extraña al poder soberano (Gregory 2004; Edkins 2000). 
Para este autor, el paradigma de la vigilancia es el campo militar, donde es posible observar un poder que actúa por el efecto de una visibilidad general: el poder se ejerce por el juego de una vigilancia exacta, y cada mirada es una pieza de funcionamiento global del poder. Una dinámica de organización que después será posible encontrar, según Foucault (1975), en la construcción de ciudades obreras, hospitales, asilos, prisiones y centros de educación, donde el principio siempre será el mismo: la organización espacial de la vigilancia jerarquizada. En cada uno de estos espacios desarrolla una arquitectura que permite distinguir un afuera y un adentro - la muralla y la barda perimetral-, haciendo posible un control interior articulado y detallado, cuya función, para quien opera esta máquina de vigilancia, es controlar y reconducir la vida de los individuos que en ella habitan o trabajan (Foucault 1975). En este sentido, la vigilancia para este autor es una de las grandes invenciones del siglo XVIII y, gracias a ella, el poder disciplinar devine en la gran maquinaria integrada - no tanto una propiedad o una cosa - que funciona como un poder múltiple, automático y anónimo que hace posible la disciplina.

Será en su análisis del modelo de panóptico propuesto por Bentham que Foucault encontrará la figura arquitectónica paradigmática que servirá como referente utópico de las instituciones disciplinarias, en el que la vigilancia sobreviene el eje central que permite la creación y conversión de cuerpos dóciles: una construcción en forma de anillo con ventanas que se orientan al interior, cada ventana no es más que una celda donde se puede colocar un loco, un enfermo o un criminal; al centro de la construcción se localiza una torre también con ventanas desde donde se puede vigilar, sin ser visto por un efecto luminoso, a los enceldados. Un dispositivo arquitectónico de este tipo hace posible experimentar con los hombres, utilizando con toda certitud las transformaciones que se pueden obtener sobre ellos (Foucault 1975).

La perspectiva de Foucault resulta significativa en la medida en que permite observar la constitución, como apunta Deleuze (2006), de una sociedad disciplinaria o de encierro, como fue la del siglo XIX y parte del XX, donde las instituciones como la escuela, la prisión, el hospital, la familia y la fábrica constituían los espacios de formación de sujetos y grupos. No obstante, para este último autor, las sociedades de encierro o disciplinarias se encuentran viviendo una crisis generalizada e irreparable después de la Segunda Guerra Mundial y están siendo sustituidas por "sociedades de control", las cuales se caracterizan por tres elementos que las distinguen de aquellas: 1) en la actualidad lo que predomina es un sistema de geometría variable cuyo lenguaje es numérico, a diferencia de la sociedad disciplinar donde los encierros funcionan más bien como moldes; 2) la emergencia de la contraseña en su versión de cifra que permite a los individuos y las poblaciones el acceso o rechazo a la información, espacios y formas de vida -los individuos han devenido dividuos-, a diferencia de las sociedades de encierro en el que predominaba la firma y que indicaba la presencia del individuo junto con el número de matrícula que establece su posición en la masa; 3) las sociedades de control funcionan como máquinas informáticas y computadoras, más que como máquinas energéticas como el panóptico (Deleuze 2006). De esta manera Deleuze imagina, por ejemplo, el futuro como un espacio fracturado por máquinas de encierro: 
[...] una ciudad en la que cada uno podía salir de su apartamento, de su casa o de su barrio gracias a su tarjeta electrónica (dividual) mediante la que iba levantando barreras, pero podría haber días u horas en los que la tarjeta fuera rechazada, lo que importa no es la barrera, sino el ordenador que señala la posición, lícita o ilícita, y que produce una modulación universal (Deleuze 2006: 284).

Para este autor, Foucault solamente retrató lo que ya no eran las sociedades contemporáneas, lo que en cierta medida dejaban de ser. Sin embargo, tal perspectiva no fue extraña a Foucault. En sus investigaciones más tardías (Foucault 2004b), este autor plantea que las sociedades modernas en general, pero en particular las contemporáneas, tienden en diferentes ámbitos de la vida social a establecer dispositivos de seguridad que buscan prevenir eventos sociales considerados como anormales o peligrosos. Para ello se echa mano del cálculo de probabilidades sobre el comportamiento humano, con el fin último de reducir lo aleatorio. Sin embargo, dichos cálculos solamente son posibles si se tiene una estrecha vigilancia de la población. Es en este sentido que la diferencia entre nuestro pasado reciente y el presente es que en las sociedades disciplinarias impera el biopoder - el control de los cuerpos individuales en los espacios cerrados-, mientras que en las sociedades de control existe una biopolítica —una vigilancia de las poblaciones en espacios abiertos. Incluso esto habla de la consolidación de la democracia, porque ella sólo puede sobrevivir en una sociedad de control, éste es el principio motor de las sociedades liberales (Foucault 2004b).

En la sociedades liberales, la vigilancia es la forma a través de la cual se establece un espacio de indeterminación o zona de excepción, en la medida en que a través de ella se controla y cuida (Lyon 1994) —con o sin autorización, eso poco importa— las acciones y comportamientos de las personas con el objeto de regular sus rutinas y hábitos, ya sea para reproducirlos o para transformarlos. La vigilancia sistemática de poblaciones, grupos e individuos, como apunta Lyon (1994), es un elemento central de la vida de los Estados-nación modernos. La emergencia de una burocracia que administra distintos aspectos de la vida (la riqueza y la pobreza, el delito y su castigo, entre otros aspectos) se incrementa incluso con la expansión de la democracia en el siglo XX. Esto resulta así en la medida en que la vigilancia es necesaria para establecer una base que responda a las demandas de igualdad y equidad, asegurando de esta forma la pertenencia de los individuos a una determinada ciudadanía y, asimismo, como una forma de control social (Dandeker 1990). Así, la vigilancia se puede convertir no sólo en un mecanismo de protección, sino también en el espacio de la regulación de la excepcionalidad.

Esto sucede cuando se analizan los dispositivos de vigilancia orientados a los pobres. Como sugiere Eubanks (2006), las nuevas tecnologías de la información tanto intensifican como facilitan la vigilancia y la disciplina en los sistemas de beneficiencia social y de políticas públicas orientadas a enfrentar la pobreza. Los diferentes instrumentos de obtención y procesamiento de información hacen factible el monitoreo de los comportamientos y rutinas vinculados a los hábitos de consumo que tienen los considerados como pobres. Por ejemplo, la utilización de tarjetas de débito donde se transfieren 
fondos a los beneficiarios de los programas de atención a la pobreza, hacen posible que se dé seguimiento de los gastos y el manejo del dinero de estos grupos, lo cual puede relacionarse con sus formas de vida y obtener perfiles de consumo que pueden determinar la permanencia o exclusión de los beneficiaros en el programa de asistencia social. Como señala la propia Eubanks (2006), con estos dispositivos de vigilancia se obtiene una reproducción de relaciones desiguales de poder y se modelan subjetividades de acuerdo a los intereses de determinados regímenes gubernamentales, en lo que se podría denominar "tecnologías de producción de ciudadanías".

Lo que se encuentra detrás de esta vigilancia hacia los pobres, como dispositivo biopolítico es, al parecer, la necesidad de construir sujetos a partir de las rutinas y los comportamientos que mantienen, conduciendo la mirada hacia el cuerpo biológico en la figura del consumo para la supervivencia, la reproducción sexual, los hábitos considerados perniciosos (como el tomar alcohol o fumar, entre otros). El pobre es concebido por estos dispositivos de vigilancia como un individuo que se encuentra en el límite de la naturaleza (la mera supervivencia biológica o vida nuda) y la vida social plena, lo cual vuelve a colocar al pobre en una zona de indistinción, entre la vida natural y el orden legal. La vigilancia, en este sentido, impone como bien apunta Giddens (1985) reglas totalitarias de organización de la sociedad. Ella permite que se justifique o no el apoyo al pobre, que se legitime su ayuda o que el pobre sea abandonado. El poder soberano, biopolítico, orienta hacia el espacio indeterminado de la pobreza una máquina de gestión de la población que, se considera, será central para la reproducción de las sociedades contemporáneas; más aún si tenemos en cuenta, como casi todos los analistas muestran, que el número de pobres seguirá en aumento, sobre todo en la región latinoamericana.

\section{EL ESPACIO JURÍDICO DE LA POBREZA: UN PROCESO QUE EXCLUYE INCLUYENDO}

Para abordar el problema de la pobreza y su situación jurídica, convendría referir al trabajo de Simmel (1998) al respecto, en la medida en que permite analizar la compleja relación que existe en las sociedades modernas entre las obligaciones y los derechos que cada ciudadano tiene con respecto a otros individuos y al conjunto social. Para Simmel, las sociedades modernas pueden comprenderse como un entramado de obligaciones y derechos. En tanto cada individuo tiene obligaciones, igualmente tiene derechos, formando con ello una red en la cual "...el derecho siempre es el elemento primero y decisivo, y donde la obligación no es, en un primer momento, más que su correlativo" (Simmel 1998:39). De esta forma, se puede considerar a la sociedad como una interacción entre derechos morales, convencionales y jurídicos. Ciertamente, unos implican obligaciones más claras que otros, y por lo tanto, castigos más o menos severos. El problema radica fundamentalmente en la definición de situaciones en las que la acción de grupos o individuos no quedan claramente definidas como producto de la obligación o del derecho. 
Una de estas situaciones que de forma paradigmática se encuentra en esta indeterminación es la de la pobreza, en particular las diversas concepciones ligadas a la obligatoriedad o no obligatoriedad de asistencia a los pobres. De entrada, para Simmel, el pobre no remite a una condición particular de carencia de bienes o servicios, sino al hecho de que pobre es aquel individuo que la sociedad —-de forma institucional 0 no- considera que debe ser sujeto de atención y ayuda. Por tanto, para Simmel, quien demanda la asistencia, el mendicante, piensa ingenuamente que tiene derecho a la caridad por su incapacidad de poder generar una riqueza personal. El derecho de los desfavorecidos de recibir un apoyo "es la base de toda noción de asistencia a los pobres" (Simmel 1998: 42).

En cierta medida, apunta Simmel, el derecho a ser asistido por estar en condición de pobreza se encuentra en la misma categoría del derecho al trabajo o el derecho a la vida: se plantea en términos de una opinión concerniente a la relación entre el individuo y los otros, entre el individuo y la totalidad de lo social, sin embargo, esto no implica que tales derechos puedan ser garantizados. Esta imposibilidad de asegurar el trabajo, la vida y el apoyo al pobre no está anclada única y exclusivamente en un elemento técnico (abrir un número determinado de empresas, asegurar las condiciones de la subsistencia de las personas, tener el dinero suficiente para proporcionar dinero y capacitación para el trabajo a los pobres), sino en la propia relación entre quien puede otorgar el derecho y quien lo puede demandar como obligación.

Efectivamente, y este el caso particular de la pobreza, en la relación de intercambio entre quien da apoyo de cualquier tipo y quien lo demanda, los pobres "...desaparecen completamente en tanto que sujetos legítimos y centros de interés” (Simmel 1998: 45). El motivo del apoyo, apunta Simmel, reside exclusivamente en la significación que toma para el donador la acción de donar. Por ello, este autor recurre a la propia enseñanza bíblica cuando Jesús conmina al rico a dar sus bienes al pobre, lo cual significa que la acción estaría orientada a la salvación de aquél, mientras que el pobre sólo constituiría un medio. Prácticamente durante el transcurso de la Edad Media esta será la interpretación canónica sobre la ayuda a los pobres: un medio que permite un fin.

Con la aparición del Estado moderno y de las instituciones que lo sustentan, entre ellas las instituciones orientadas a tender o resolver el problema de la pobreza, éstas presentan en cierta medida una característica análoga al situar en el pobre no el fin sino el medio para algo más. Paradójicamente, apunta Simmel, son instituciones que se concentran en la actividad concreta del individuo y su situación social. A diferencia de la armada, la policía, las escuelas, los trabajos públicos y la administración judicial, que están dirigidas hacia la totalidad de los individuos, aquellas orientadas a la atención a los pobres consideran a éstos como individuos diferentes de otros y por ello requieren de una observación constante: el individuo pobre, en su acepción moderna, "representa el último eslabón, pero ciertamente no el objetivo final, que consiste únicamente en proteger y mantener la comunidad en su lugar" (Simmel 1998:48). Los pobres no pueden ser considerados incluso, refuerza Simmel a continuación, como los medios de un fin, lo cual sería ya un progreso en su situación, pues la acción institucional no los toma en 
cuenta ni siquiera en tanto individuos, así no utiliza más que ciertos objetivos y recursos a fin de suprimir los peligros y las pérdidas que representan ellos con relación al bien de la comunidad (Simmel 1998)2.

De esta forma, para Simmel la asistencia y el apoyo a los pobres tiene como meta mitigar las manifestaciones extremas de diferenciación social, a fin de que la sociedad pueda seguir estructurada sobre tales diferencias. De hecho, en la actualidad el Estado, pese a las transformaciones recientes que lo retiran cada vez más de la esfera de la asistencia social, tiene bajo su encargo el prestar ayuda a los pobres, pero ello no fuerza a generar una obligación en términos legales. Simmel señalaba atinadamente que el Estado no reconoce en la figura social de la pobreza la obligación de asistencia, ya que ninguno de los grupos y sujetos localizados en esta categoría tiene algún recurso jurídico para acceder a la asistencia. De hecho, y quizás esto es lo más importante, pueden recibirla pero no reivindicarla. Esta conclusión resulta reveladora en la reflexión de Simmel porque lleva a considerar finalmente que,

[... en el seno del Estado moderno relativamente democrático, la asistencia pública es probablemente el único espacio de la administración en la que los interesados no tienen alguna participación. [...] La asistencia a los pobres es en efecto una aplicación de medios públicos con fines públicos; y dado que los pobres se encuentran excluidos de esta teleología [...] la relación entre derecho y obligación puede ser modificada (Simmel 1998: 52-53).

Esto representa, en última instancia, la exclusión de los pobres de una cadena teleológica -cadena que implica la aplicación de medios públicos con fines públicos-, lo que les impide constituir tanto un medio como un fin. Se crean instituciones públicas para asistir a los individuos pobres en sus distintas necesidades, a través de programas y políticas públicas, pero los individuos en situación de pobreza no pueden exigir ser beneficiados por esas políticas porque no tienen el derecho de reclamar asistencia. Esto representa un problema porque de entrada, dice Simmel, los pobres son también ciudadanos, tienen derechos y obligaciones. En ese sentido, aunque sea en términos abstractos, el Estado tiene la obligación de asistirlos. No obstante, los pobres son asistidos no por ser ciudadanos, sino por el rol que juegan en las estrategias que permiten al Estado mantener la estructura de desigualdad social y, con ello, la estabilidad social. Esta situación coloca a los pobres en una posición particular como ciudadanos frente al Estado, ya que son a la vez: "el objetivo externo de un acto de asistencia y un objeto inerte disminuido en sus derechos en los objetivos globales del Estado" (Simmel 1998: 55). De esta forma, apunta Simmel, los pobres están colocados fuera del Estado (no pueden reclamar "derechos de pobres"), pero están también integrados a él de forma

\footnotetext{
${ }^{2}$ Lo cual coincide con lo que dice Foucault acerca de las sociedades liberales: la politica social en estas sociedades refiere, sobre todo, a las cuestiones de seguridad (Foucault 2004a).
} 
orgánica. Esto, concluye Simmel, significa que los pobres están “...en la situación del extranjero que se encuentra por decirlo de alguna manera materialmente fuera del grupo en el que reside" (Simmel 1998: 56).

En este sentido, la posición del pobre es la de un ciudadano que se encuentra en una situación donde el Estado lo excluye incluyéndolo. Los pobres son de entrada ciudadanos, y por ello presentan una relación de obligaciones y derechos frente a otros ciudadanos y al Estado. Sin embargo, su posición de carencia los excluye de la relación con otros ciudadanos en condiciones distintas y también los ubica en una posición diferente con respecto al Estado. No pueden exigir de éste, en términos legales, apoyo para la satisfacción de sus demandas, aunque moralmente consideren que sí. Por otro lado, el apoyo que llegan a recibir no está orientado, como finalidad, a satisfacer sus necesidades, sino a cumplir con una teleología diferente: legitimar los mecanismos de desigualdad social. Sin embargo, lo más importante de la reflexión llevada a cabo por Simmel sobre los pobres están —al menos para los objetivos de este documento- en la forma en como el Estado establece de forma arbitraria el apoyo a los considerados como pobres.

Para Simmel, como se ha visto, es en el Estado donde recae el reconocimiento de quién es pobre y, además, de quién debe recibir el apoyo. De esta forma, el Estado deja a un lado la relación de reciprocidad de una red de derechos y obligaciones frente al ciudadano, generando con ello un espacio de excepción: el Estado se coloca, al menos en este punto, por encima de cualquier normatividad y ley, pues es éste quien decide la excepción. Tal situación, sin embargo, no responde a un problema localizado en una minucia de carácter legal, sino que se encuentra en las propias bases que sustentan al Estado occidental, ya que éste es el único con la capacidad para decidir la excepción, es decir, de colocarse fuera de la ley para tratar de reestablecer la ley y la norma frente aquello que sólo puede alcanzar suspendiéndose, es decir, la vida. Retomando el concepto acuñado por Carl Schmitt (1988), esta capacidad de decidir la excepción es la soberanía. Consecuente con esto, conviene explorar la reflexión que en este sentido lleva a cabo Agamben (2006), pues este autor señala que cuando el sistema de responsabilidades legales se suspende produce espacios de excepción (Gregory 2004). A partir de esto, se explora la paradoja encontrada ya en la definición de pobreza ofrecida por Simmel, así como la serie de mecanismos echados a andar por los Estados moderno para atenderla, denotados en el recurso de tecnologías de vigilancia.

\section{Pobreza COMO INDETERMINACIÓN: LA FIGURA DE LA VIDA NUDA}

Agamben (2006) considera que "la política occidental ha sido biopolítica desde sus inicios": es en la toma de la vida por el poder político donde encontramos desde siempre la fuente de la soberanía en el mundo occidental, desde la Roma clásica hasta nuestros días. Desde esta óptica el poder es biopoder desde el principio y sólo podríamos hablar de soberanía y poder político en la era moderna si de inicio relacionamos la vida con 
ambos conceptos. De hecho, apunta Agamben (2006), la concepción contemporánea de la soberanía, acuñada en gran medida por Carl Schmitt (1988), se basa en el hecho de que el soberano es simplemente "quien decide la excepción". En otras palabras, el soberano es el único que puede decidir cuándo la ley se suspende. Tal definición implica que si solamente el soberano puede decidir la excepción, entonces está más allá de la ley, es decir, domina aunque la ley haya sido suspendida. En este sentido, el soberano es anterior y posterior a la ley.

El beneficio que obtiene el soberano al estar a la vez antes y después de la ley es la extensión de su poder, abarcando lo que el derecho contempla y, esto es quizás lo más importante, lo que la ley no puede abarcar ni contemplar. Lo que está afuera de la ley bien puede ser lo que en la filosofía política clásica se considera como el "estado de naturaleza", figura que en buena medida es equivalente a la vida nuda, la vida más allá de la ley. Efectivamente, al parecer, la vida nuda se encuentra en un espacio donde la ley suspende su capacidad de norma porque no puede o no debería establecer mecanismos para su regulación, en la medida en que es la vida misma ${ }^{3}$. Sin embargo, eso que al parecer está antes de la ley, lo que queda una vez suspendida la ley, no es algo totalmente excluido, no es algo que se encuentre irremediablemente en el fuera; bien al contrario, es aquello que por este gesto de exclusión de la ley está incluido bajo el dominio del poder soberano, siempre bajo un proceso de exclusión incluyente. Otra manera de llamar este gesto ambiguo de exclusión incluyente es el "abandono". Aquello que el poder soberano excluye queda de alguna manera en "estado de abandono", una situación donde se deja a su suerte a las personas y su vida, un lugar sin ley, sin estatus jurídico, pero sometido totalmente al poder soberano. Agamben (2003) no duda en pensar en los detenidos de Guantánamo o en los judíos de los campos de concentración alemanes como las figuras extremas de este abandono: se encuentran en un lugar sin ley, sin derechos y obligaciones, pero sometidos al poder soberano, que decide su suerte: la excepción o la aplicación de la ley.

Esta figura "abandonada" puede ser la del pobre. Ciertamente no es el mismo caso del judío en el campo de concentración o el supuesto terrorista islámico en Guantánamo, no obstante también se encuentra habitando un espacio de excepción. El Estado, como poder soberano, decide, como apuntamos más atrás siguiendo a Simmel, quién es pobre, qué tipo de ayuda debe recibir y hasta cuándo. El pobre no puede reclamar ningún tipo de ayuda porque si bien es ciudadano, no tiene un estatus jurídico que le permita apelar a una cierta ayuda por su condición de carencia. Su situación lo pone en un estado de abandono, cercano al estado de naturaleza o, para considerarlo de forma más contemporánea, en la condición de exclusión incluyente. Ya que el pobre, por un lado, está excluido del circuito de derechos que le permiten reclamar legalmente asis-

\footnotetext{
${ }^{3}$ Recordemos que para Agamben (2006) lo que está antes del contrato, de la fundación del Estado, es la vida nuda, la vida en su estado de naturaleza o zoé, misma que al conformarse el contrato o la vida dentro de la ley, debe quedar fuera.
} 
tencia; pero es precisamente esta situación de exclusión lo que lo introduce más fuertemente en el circuito de la vigilancia por parte del Estado, a través de la asistencia. Este doble proceso de exclusión-inclusión que se produce al mismo tiempo es, siguiendo las reflexiones de Agamben (2006; 2003), lo que coloca a los pobres en una zona de excepción. El mundo moderno, de hecho, y la pobreza no es su único ejemplo, el lugar de ser "excepcional" se convierte en la regla: nos encontramos ante la pululación y normalización de pequeños estados de excepción en nuestra vida cotidiana, en la calle, en el trabajo, en la familia.

Sin embargo, podemos considerar que el manejo del llamado "problema de la pobreza" y su "combate" — nótese siempre la presencia, cuando se habla del tema, de un cierto tono "guerrero", propio de los estados de excepción- es una de las mejores muestras de cómo actúa el poder soberano en nuestros días y, con él, el estado de excepción. La atención a la pobreza no produce cuerpos excluidos, sino el gesto ambiguo de lo que al ser excluido queda incluido, cuerpo abandonado que desposeído por la ley tendrá que quedar sujeto al poder total del soberano y a su posible asistencia.

Así, el cuerpo fabricado por los discursos y las políticas de "combate a la pobreza" no es un cuerpo excluido, sino un cuerpo abandonado, incluido en virtud de su exclusión, un cuerpo sin estatus jurídico, fuera de la ley y por ello mismo, totalmente abandonado al poder soberano. "Ban", prefijo del cual surgen la palabra abandono (en inglés, banishment, en francés bannir), puede encontrarse ya en el Código de Hammurabi y más adelante en La Política de Aristóteles. Como apunta Calarco el "ban — sea en términos de banishment, abandono, exilio u ostracismo - lejos de ser un mero castigo por un crimen, se utiliza cuando la vida de un individuo se considera virulenta para la comunidad" (2007: 77).

En cierto aspecto, para Agamben (2006), aquello que se considera peligroso para la comunidad no puede dejarse al azar de la pura exclusión, del puro afuera, y tiene más bien que ser introducido en un régimen de poder, a la vez que es excluido del orden legal. Ahora bien, tal espacio, que al excluir incluye, no está ni en el adentro ni en el afuera, sino en una "zona de indistinción", habitada por cuerpos reducidos a la vida nuda, estrato biológico que paradójicamente queda politizado al entrar bajo el régimen soberano. La vida nuda, producto de tal zona de indistinción, de la cual el cuerpo abandonado sería una de sus expresiones, es en última instancia aquello que permanece sumiso al poder soberano antes y en la ausencia de la ley. En este talante, el cuerpo fabricado por los discursos y las políticas de la pobreza es vida nuda, cuerpo abandonado que en virtud de su exclusión del orden legal es conducido con mayor firmeza dentro del poder soberano.

De esta manera, el cuerpo del pobre no es un cuerpo meramente excluido, es más bien un cuerpo abandonado, incluido en virtud de exclusión. Cuerpo sumiso por estar a la vez dentro y fuera, dentro del poder soberano, fuera de la ley. En esta lógica, esta situación expresa dos movimientos que se apoyan mutuamente, pero que son contradictorios: inclusión, por un lado, maximización de la vida; exclusión, por el otro, derecho a dejar morir por abandono, por falta de apoyo o de asistencia al pobre. Éste no está ni adentro ni afuera, no está ni meramente excluido ni meramente incluido, se encuentra 
antes bien en una zona de indistinción que lo incluye al estar sometido a un poder total, a la vez que lo excluye de todo derecho, de toda existencia legal.

De esta forma, aquello que Simmel considera un problema entre el Estado y su definición con respecto a los derechos de los pobres, sólo muestra de forma exacerbada la propia condición de soberanía del Estado moderno, que implica la capacidad de ejercer una biopolítica y necropolítica de la población ${ }^{4}$. En este sentido, el problema de qué hacer con los pobres, si apoyarlos o abandonarlos, permite observar con mayor claridad la capacidad de construir espacios de excepción jurídica que abren camino a la gestión de la vida nuda. Sin embargo, el poder soberano necesita establecer de alguna manera los contornos que delimitan las zonas de indistinción. Por ello recurre, como el propio Agamben (2007) señala, a dispositivos de gubernamentalidad: maquinarias de vigilancia de la población y de los individuos.

Los sistemas de vigilancia electrónicos de hoy en día ponen un acento particular a estas formas de control, orientación y modelación de sujetos, asunto apuntado de alguna manera por Foucault (quién logró definir algunos de estos dispositivos en sus trabajos sobre la noción disciplina). No obstante, no sólo la prisión, la escuela, el asilo y el hospital son dispositivos. Siguiendo a Agamben (2007), sería necesario incluir también a los ordenadores, a los teléfonos celulares, a las cámaras de vigilancia, a los dispositivos biométricos y electrónicos, ya que ellos abren nuevas formas de subjetivación y desubjetivación, y en el caso de la pobreza, tales dispositivos cumplen un papel importante.

\section{DISPOSITIVOS DE POBREZA COMO NECROPOLÍTICA}

La indeterminación jurídica del pobre responde, como se ha visto, al carácter en el cual se funda la soberanía del Estado moderno, sobre todo por su carácter a la vez de incluido y excluido: incluido en procesos de vigilancia y gestión; excluido de un pleno reconocimiento de sus derechos. La figura del pobre es una forma particular —quizás la más significativa - de la construcción de espacios de excepción, donde los individuos se encuentran permanentemente vigilados, incluso, no sólo por su condición de carencia

\footnotetext{
${ }^{4}$ Cabe señalar que Agamben (2006) utilizó una connotación cercana a la noción de necropolítica para exponer la gestión de la muerte, señalando una tanatopolítica. La diferencia entre ambos conceptos y autores, partiría de la etimología griega con la que forjan dicha terminología: tanatos refiere a la figura de la muerte como persona, como sustantivo; nekros, a la cualidad de la muerte, al adjetivo. De este modo, la diferencia radicaría en quién tiene la decisión de dar muerte, la decisión sobre el estado de excepción y la calidad de la muerte. En el caso de la tanatopolítica, ésta implica la definición de la "vida digna de vivirse" (Agamben, 2006: 156), es decir, implica la decisión soberana sobre el umbral biopolítico. En otro sentido, la necropolítica, define el estatus de "muerto viviente" aplicado a las poblaciones (Mbembe, 2003: 40). Así, el concepto de Mbembe (2003) resulta una profundización de aquel descrito por Agamben. Sobre este aspecto los autores agradecen los atinados comentarios y sugerencias al respecto realizados por Roberto Fuentes Rionda. Para una discusión sobre este punto ver Gregory (2004).
} 
económica, sino porque el pobre también entrañaría al potencial delincuente, un "salvaje" que carece de los soportes que le permiten integrarse plenamente a la sociedad. En otras palabras, ésta es la figura contemporánea más extendida de la vida nuda, que significa vida gestionada y vigilada.

Paradójicamente, por lo general, las distintas políticas orientadas al "combate" o "lucha" contra la pobreza tratan de transformar las prácticas y rutinas de la vida de los considerados como pobres, con el fin de incluirlos en lo social. No obstante, este mecanismo resulta ser doble: pretende excluir incluyendo. Nada regula esta relación, pues el Estado es quien establece no sólo quién es pobre, sino quién de entre ellos merece recibir algún apoyo. Los pobres, en cuanto tales, jamás podrán reclamar la ayuda por su condición, como ya lo ha mostrado Simmel. Esto puede observarse en el conjunto de América Latina, donde la gran parte de sus estados, impulsan variadas estrategias de ayuda 0 apoyo contra la pobreza, pero en cada caso representan el ejercicio de una biopolítica erigida en una serie de dispositivos de gubernamentalidad y subjetivización que implican un grado creciente en la aplicación de dispositivos de vigilancia.

De esta forma, un dispositivo de vigilancia es una máquina que produce subjetividades y, al mismo tiempo, desubjetiviza, por lo que siempre es una máquina de gobierno en tanto tiene "la capacidad de capturar, orientar, determinar, interceptar, modelar, controlar y asegurar los gestos, las conductas y las opiniones" de las personas (Agamben 2007: 31). En el capitalismo contemporáneo, más que en ninguna otra época, los dispositivos generan no sólo sujetos, sino procesos de desdibujamiento de los mismos. En tal sentido, no es posible hablar más de un buen uso o mal uso de los dispositivos, pues de entrada son máquinas de gobierno que transforman y reproducen sujetos, tanto para bien como para mal. En este sentido, en mayor o menor medida las sociedades actuales:

[...] introducen hacia todos los ciudadanos esos dispositivos biométricos que perfeccionan las tecnologías antropométricas (desde la impresión de las huellas digitales hasta las fotografías signaléticas) que habían sido inventadas en el siglo XIX para identificar a los criminales reincidentes, la video vigilancia transforma los espacios públicos de nuestras ciudades en interiores de inmensas prisiones (Agamben 2007: 48).

En el caso de la pobreza, se pueden observar mecanismos de control de la vida con el fin de crear espacios de exclusión. Las favelas, las ciudades perdidas y todos esos eufemismos refieren al espacio donde la vida ha sido reducida por el Estado o donde éste dispone a reducirla a la mera reproducción de la vida biológica. En cierta forma, ésta es una forma de control y subyugación de la vida -y de la muerte- a la capacidad y al poder, que transforma los mecanismos que construyen los sujetos. En este sentido, sobre el considerado pobre se ejerce una particular necropolítica, como apunta Mbembe (2003), y no sólo una biopolítica, en la medida en que se domina la vida por el camino de la gestión de la muerte. Esto se puede observar, por ejemplo, en la construcción del discurso minimalista sobre la pobreza, del cual da cuenta ampliamente Álvarez (2005), 
en el que se busca determinar un posible ingreso "mínimo" para los pobres, condiciones "mínimas" para la población, la canasta "básica" para la supervivencia5. Éstos, entre otros términos, tienden a poner como telón de fondo de las políticas neoliberales el supuesto de un grupo de seres humanos que pueden vivir con sólo encontrarse en el umbral de su reproducción como seres biológicos.

Las llamadas políticas de combate a la pobreza evidencian la existencia clara de una necropolítica en los estados poscoloniales (Mbembe 2003), a través del cual se desarrollan varios y diversos dispositivos que pretenden definir a dichos grupos humanos únicamente mediante nuevas y únicas formas de existencia social, logradas gracias a un cierto estatus de muertos vivientes (Norris, 2000), semejantes a habitantes de mundos muertos (Mbembe 2003) -especie de zombies que, equivalentes a los personajes de las secuelas cinematográficas de George Romero, son constantemente excluidos por los "vivos" de diferentes espacios: zonas habitacionales dignas, escuelas, centros comerciales, así como de cualquier forma de consumo más allá del referente básico al que se les sujeta. Los pobres se convierten en objetos de una soberanía estatal que funda sus bases, precisamente en su capacidad de decidir entre quién vive y quién muere ${ }^{6}$.

El despliegue, poco más, poco menos, de la totalidad de los instrumentos del Estado en América Latina en la extensión del espacio de indeterminación que representa la pobreza, con el fin de vigilar a los pobres, es una demostración tersa y lenta del poder político sobre la vida cotidiana de los pobres. A esto habría que agregar que, en el transcurso del día a día, estos sectores subsisten en una especie de "zona de poder salvaje" (Buck-Morss 2003), caracterizada por la violencia y la precariedad, en la que se pueden registrar altos índices de muerte infantil, violencia intrafamiliar, mala nutrición; viviendo bajo un clima de profundo racismo, en espacios donde la violencia política, e incluso de grupos paraestatales, generan un ambiente en el que impera la sensación de hallarse dentro de un espacio con un sostenido poder basado en la gestión y la producción de muerte.

\footnotetext{
${ }^{5}$ En el caso latinoamericano, las políticas sociales de "combate" a la pobreza han tenido un desarrollo histórico aparejado con las estrategias económicas. De este modo, durante la última década, al incorporarse a la lógica de la globalización y al neoliberalismo, los Estados latinoamericanos han puesto en marcha estrategias sociales orientadas a la potencialización de aptitudes y competencias económicas, proveyendo a la población identificada como pobre, suponen, los medios para salir de su situación, basados en la presunción de un límite necesario para la subsistencia de los individuos. Así, las políticas sociales que refieren a la pobreza en América Latina se han sustentado en ingresos económicos mínimos para cubrir necesidades mínimas, partiendo en mecanismos de libre competencia Muchas de estas políticas han sido diseñados desde los órganos internacionales como el Banco Interamericano de Desarrollo (BID), por lo que no se puede dejar de un lado el papel que han jugado los organismos multilaterales en el diseño de las políticas de focalización y el "universalismo básico" (ver: Arteaga, 2006, 2005a, 2005b, 2003; Bolvitnik y Hernández Laos, 2006; Neffa 2005; Queiroz Ribeiro, 2005; Székely, 2005; Superveille y Quiñones, 2005).

${ }^{6}$ De hecho, siguiendo a Agamben (2006), al localizarse a los pobres en una situación de vida nuda, pueden por esa misma causa ser objeto de ayuda y protección.
} 


\section{CONCLUSIONES}

Por ello, los dispositivos de vigilancia de la pobreza juegan un papel central en la redefinición de las sociedad en América Latina, pues son guiados por el espíritu de mantener, a buen resguardo, los espacios de indeterminación de los ciudadanos considerados como pobres: organizan grupos y espacios sociales, en otras palabras, definen un proyecto específico de sociedad (Donzelot 2003); pero además, garantizan la administración de los pobres en lo que bien se podría llamar una micro-gestión de la carencia, produciendo, con ello, una específica micro-política de la muerte. De ahí la importancia, consideramos, de una reflexión como la que se ha intentado sontener aquí: la microgestión de la pobreza deberá permitir pensar el ejercicio de la política como una manera de instrumentalizar la existencia humana, a través de la puesta en marcha de dispositivos específicos, cuyo fin sería la destrucción, como sugiere el propio Mbembe (2003), de los cuerpos humanos y de las poblaciones -considerando en particular en América Latina, pero de igual forma en otros continentes, y que no se reducen a esos espacios marcados por la guerra, el genocido y los campos de concentración.

Finalmente, lo que hemos querido destacar en este artículo es la profunda imbricación entre el estatus de la pobreza tal como es tratada y manejada por el Estado, en particular en las sociedades latinoamericana actuales, la cual de inicio está excluida de un reconocimiento jurídico pleno, y la manera como esta exclusión de pie a una inclusión más decidida de los pobres dentro del campo de aplicación del poder soberano a través de programas de apoyos, subsidios y otros mecanismos de vigilancia-asistencia, mismos que el Estado puede decidir, sin obligación legal alguna, suspender o sostener. Tal estado de exclusión incluyente arroja al pobre a una verdadera zona de indistinción en la cual está a la vez dentro y fuera de la ley: fuera, en tanto su situación de precariedad no puede dar lugar a una demanda legalmente reconocida; dentro, en tanto objeto de políticas de asistencia que terminan apoyándose en dispositivos de vigilancia y seguimiento biopolítico.

\section{REFERENCIAS BibLIOGRÁFICAS}

Agamben, G. 2006. Homo Sacer. El poder soberano y la nuda vida. Valencia: Pre-textos.

Agamben, G. 2003. État d'exception. Homo Sacer II, 1. Paris: Éditions du Seuil.

Agamben, G. 2007. Qu'est-ce qu'un dispositif?. Paris: Rivages Poche.

Álvarez Leguizamón, Sonia. 2005. "Los discursos minimistas sobre las necesidades básicas y los umbrales de ciudadanía como reproductores de la pobreza." Pp. 239-273, en Trabajo y producción de la pobreza en Latinoamérica y el Caribe: estructuras, discursos y actores compilado por Sonia Álvarez Leguizamón. Buenos Aires: CLACSO-CROP. 
Barajas, Gabriela 2002. "Las Políticas de atención a la pobreza en México, 1970-2001: de Populistas a Neoliberales." Revista Venezolana de Gerencia 20: 553-578.

Bialakowski, A. L., Ana L. López e M. M. Patrouilleau 2007. "Práticas governamentais na regulamentação de populações extinguíveis." Pp. 151-194 en Produçao de pobreza e desigualdade na América-Latina organizado por Alberto Cimadeamore y Antonio Catani. Porto Allegre: CLACSOCROP-TOMO-UFRGS.

Arteaga Botello, Nelson 2003. "El abatimiento de la pobreza en México (2000-2006)." Pp. 139-166 en Pobreza urbana, perspectivas globales, nacionales y locales, Miguel Ángel Porrúa, coordinado por Nelson Arteaga Botello et. al. México. Gobierno del Estado de México: CEMAPEM.

Arteaga Botello, Nelson 2005a. "The future that will not come: the eradication of poverty from the Mexican federal government's viewpoint (2000-2006)." Pp. 135-155 en The Poverty of the State. Reconsidering the role of the state in the struggle against global poverty, editado por Alberto Cimadamore et. al. Buenos Aires: CLACSO-CROP / NORAD.

Arteaga Botello, Nelson 2005b. "Los eternos excluidos: pobreza y desarrollo social desde una perspectiva socio-histórica. (El caso de tres comunidades del municipio de Toluca)." Pp. 49-69 en Pobreza, empleo y participación ciudadana: aportes para la política social, coordinado por Raúl López Estrada et. al. México: Universidad Autónoma de Tamaulipas, Universidad Autónoma de Nuevo León.

Arteaga Botello, Nelson 2006. Pobres y delincuentes. Estudio de sociología y genealogía. México: Miguel Ángel Porrúa / Cámara de Diputados LIX Legislatura / FCPyAP / UAEM.

Boltvinik, Julio y Enrique Hernández Laos 2006. Pobreza y distribución del ingreso en México. México: Siglo XXI.

Buck-Morss, Susan 2003. Thinking past terror: Islamism and critical theory on the left. Londres: Verso.

Cimadamore, Alberto, Hartley Dean y Jorge Siqueira 2005. Poverty of the state: reconsidering the role of the state in the struggle against global poverty. Buenos Aires: CLACSO.

Deleuze, Gilles 2006. Conversaciones. Valencia: Pre-textos.

Donzelot, Jacques 1984. L'invention du social. Essai sur le décline des passions politiques. París: Éditions du Seuil.

Duffield, M. 2002. "Social reconstruction and the radicalisation of the development: aid as a relation of global liberal governance." Development and Change. 33: 1049-1071.

Edkins, Jenny 2000. "Sovereign power, zones of indistinction and the camp." Alternatives 25: 3-25.

Eubanks V. 2006. "Technologies of Citizenship: Surveillance and Political Learning in the Welfare System." Pp. 89-107 en Surveillance and security: Technological politics and power in everyday life, editado por Torin Monahan. USA: Routledge.

Foucault, Michel 1976. Histoire de la sexualité I. La Volonté de savoir. París: Gallimard.

Foucault, Michel 2004a. Naissance de la biopolitique (Cours au Collège de France, 1978-1979). Paris: Seuil. 
Foucault, Michel 2004b. Securité, territoire, population. París: Gallimard.

Foucault, Michel 1975. Surveiller et punir. Naissance de la prision. París: Gallimard.

Giddens, Anthony 1985. The Nation-State and violence. Cambridge: Polity Press.

Gregory, Derek 2004. The colonial present. Oxford: Blackwell.

Mbembe, A. 2003. "Necropolitics". Public Culture 15: 11-40.

Neffa, Julio César 2005. "Pobreza y producción de la pobreza en Latinoamérica y el Caribe." Pp. 193208 en Trabajo y producción de la pobreza en Latinoamérica y el Caribe: estructuras, discursos y actores, compiladora Sonia Álvarez Leguizamón. Buenos Aires: CLACSO-CROP.

Norris, Andrew 2000. "Giorgio Agamben and the politics of the living dead." Diacritics 30: 38-58.

Queiroz Ribeiro, Luiz César, 2005. "Segregación residencial y segmentación social: el 'efecto vecindario' en la reproducción de la pobreza en las metrópolis brasileñas." Pp. 137-156 en Trabajo y producción de la pobreza en Latinoamérica y el Caribe: estructuras, discursos y actores, compiladora Sonia Álvarez Leguizamón. Buenos Aires: CLACSO-CROP.

Sandbrook, R. y Romano D. 2004. "Globalization, extremism and violence in poor countries." Third World Quaterly 25: 1007-1030.

Székely, Miguel. 2005. Desmitificación y nuevos mitos sobre la pobreza. México: Miguel Ángel Porrúa/ Ciesas.

Supervielle, Marcos y Mariela Quiñones. 2005. "De la marginalidad a la exclusión social: cuando el empleo desaparece." Pp. 99-136 en Trabajo y producción de la pobreza en Latinoamérica y el Caribe: estructuras, discursos y actores, compiladora Sonia Álvarez Leguizamón. Buenos Aires: CLACSO-CROP.

Schmitt, Carl. 1988. Théologie politique. París: Gallimard.

NELSON ARTEAGA BOTELLO es Doctor en Sociología. Profesor investigador de la Facultad de Ciencias Políticas y Sociales de la Universidad Autónoma del Estado de México. Línea de investigación: Violencia, pobreza y dispositivos de vigilancia. Publicaciones recientes: "An Orchestration of Electronic Surveillance: A CCTV Experience in Mexico", en International Criminal Justice Review, (17) 4: 325-335, (2007).

MARÍA LUISA BACARLETT PÉREZ es Doctora en Filosofía. Artículos más destacados: "Georges Canguilhem. Una filosofía de lo biológicamente posible" (2006) y "Canguilehm y Foucault: de la normatividad a la normalización" (2009), ambos en la Revista Ludus vitalis. Libros más importantes: Friedrich Nietzsche: la vida, el cuerpo y la enfermedad (2006) y Filosofía y enfermedad: un acercamiento a la obra de Georges Canguilhem.

RECIBIDO: $15 / 10 / 2008$

ACEPTADO: 8/08/2009

Publicado on-line: 28 de abril de 2010 